\title{
El profesor como guía y guerrero: metáforas sobre la profesión docente*
}

\author{
PAOLA ALARCÓN HERNÁNDEZ** \\ VÍCTOR VÁSQUEZ BUSTOS *** \\ CLAUDIO DÍAZ LARENAS**** \\ CAROLINA VENEGAS CARRASCO ${ }^{* * * * *}$
}

Recepción: 10 de marzo de 2020

Aprobación: 20 de junio de 2020

Forma de citar este artículo: Alarcón, P., Vásquez, V., Díaz, C. \& Venegas, C. (2020). El profesor como guía y guerrero: metáforas sobre la profesión docente. Cuadernos de Lingüística Hispánica, (36), 105-128.

https://doi.org/ 10.19053/0121053X.n36.2020.10989

* $\quad$ Artículo de investigación. Investigación patrocinada por los proyectos FONDECYT Regular N. ${ }^{0}$ 1150889: "Las dimensiones cognitivas, afectivas y sociales del proceso de planificación de aula y su relación con los desempeños pedagógicos en estudiantes de práctica profesional y profesores nóveles de pedagogía en inglés"; y FONDECYT de Iniciación N. ${ }^{0} 11130482$ : "La metáfora conceptual como herramienta de análisis de creencias docentes en profesores de establecimientos educacionales de la comuna de Concepción".

** Profesora de Español, Doctora en Lingüística, Universidad de Concepción, Chile; profesora asociada, Departamento de Español, Facultad de Humanidades y Arte, Universidad de Concepción, Chile. Correo electrónico: palarco@udec.cl ®https:// orcid.org/0000-0001-7217-5668.

*** Profesor de Español, Magíster en Lingüística Aplicada, Universidad de Concepción, Chile. Correo electrónico: victorpatriciovb@ gmail.com ㄴhttps://orcid.org/0000-0003-1334-9896

**** Profesor de Inglés, Doctor en Educación, Universidad de Concepción, Chile; profesor titular, Departamento de Currículum e Instrucción, Facultad de Educación, Universidad de Concepción. Correo electrónico: claudiodiaz@udec.cl Đhttps://orcid. org/0000-0003-2394-2378

***** Psicóloga, Magíster en Investigación Social y Desarrollo, Universidad de Concepción, Chile; profesora asistente, Departamento de Salud Pública, Facultad de Medicina, Universidad de Concepción, Chile. Correo electrónico: carovenegasc@udec.cl @https:// orcid.org/0000-0002-4091-2974 


\section{Resumen}

En el contexto de las diferentes reformas al sistema educacional chileno y de las movilizaciones que han suscitado, el objetivo de la investigación es analizar los conceptos metafóricos sobre la figura del profesor. En el estudio se empleó un enfoque cualitativo y se adoptó un diseño fenomenológico. Se aplicó un cuestionario de obtención de metáforas a 168 estudiantes de los cursos finales de pedagogía en inglés de universidades chilenas. El análisis de las expresiones metafóricas obtenidas se efectuó mediante un análisis de contenido semántico. A partir del análisis, se establecieron tres categorías mayores: metáforas centradas en el profesor, metáforas centradas en el estudiante y metáforas centradas en el reconocimiento social del profesor. Los resultados muestran que, aunque los participantes están conscientes de las dificultades de su ejercicio, tienen una valoración positiva de la profesión docente. Paralelamente, predominó la visión del profesor como un mediador en el aprendizaje de sus alumnos.

Palabras clave: investigación cualitativa, estudiantes de pedagogía, análisis del discurso, metáforas.

\section{The Teacher as Guide and Warrior: Metaphors English Teachers about the Teaching Profession}

\section{Abstract}

In the context of various reforms to the Chilean educational system and the demonstrations that it has provoked, the objective of this investigation is to analyse the metaphorical concepts about the figure of the teacher. In the study we used a qualitative approach and a phenomenological design. 168 Chilean English Teaching students who were in the final years of their undergraduate degree answered an eliciting metaphor questionnaire. The analysis of the metaphorical expressions obtained from the questionnaire was carried out through semantic content analysis. From this analysis, three major categories were defined: metaphors focused on the teacher, metaphors focused on the students and metaphors focused on the teacher's social recognition. The results show that, even though the subjects are aware of the difficulties of their practice, they have a positive impression of the teaching practice. In parallel, the prevailing idea was that of the teacher as a medium for the learning of his or her students.

Keywords: qualitative research, pre-service teachers, discourse analysis, metaphors. 


\section{Le professeur comme guide et guerrier : métaphores sur la profession enseignante}

\section{Résumé}

Dans le contexte des différentes réformes du système éducatif chilien et des mobilisations qu'elles ont suscitées, l'objectif de la recherche est d'analyser les concepts métaphoriques de la figure du professeur. L'étude a adopté une approche qualitative et une conception phénoménologique. Un questionnaire d'obtention de métaphores a été appliqué à 168 étudiants des derniers cours de pédagogie en anglais des universités chiliennes. L'analyse des expressions métaphoriques obtenues a été effectuée au moyen d'une analyse sémantique. À partir de cette analyse, trois grandes catégories ont été établies: métaphores centrées sur le professeur, métaphores centrées sur l'étudiant et métaphores centrées sur la reconnaissance sociale del professeur. Les résultats montrent que, bien que les participants soient conscients des difficultés de leur exercice, ils ont une appréciation positive de la profession enseignante. Parallèlement, la vision de l'enseignant comme médiateur dans l'apprentissage de ses élèves a prévalu.

Mots-clés: recherche qualitative, étudiants en pédagogie, analyse du discours, métaphores.

\section{0 professor como guia e guerreiro: metáforas sobre a profissão docente}

\section{Resumo}

No contexto das diferentes reformas do sistema educacional chileno e das manifestações que suscitaram, o objetivo da investigação é analisar os conceitos metafóricos sobre a figura do professor. No estudo utilizou-se um enfoque qualitativo e adotou-se um desenho fenomenológico. Um questionário de obtenção de metáforas foi aplicado a 168 estudantes dos cursos finais de pedagogia em inglês de universidades chilenas. A análise das expressões metafóricas obtidas efetuou-se por meio de uma análise de conteúdo semântico. A partir da análise, se estabeleceram três categorias maiores: metáforas centradas no professor, metáforas centradas no estudante e metáforas centradas no reconhecimento social do professor. Os resultados mostram que, apesar de que os participantes estão conscientes das dificuldades do seu exercício, tem uma valorização positiva da profissão docente. Paralelamente, predominou a visão do professor como um mediador na aprendizagem dos seus alunos.

Palavras-chave: investigação qualitativa, estudantes de pedagogia, analise do discurso, metáfora. 


\section{Introducción}

Durante la última década en Chile se ha implementado una serie de reformas que regulan el ejercicio de la profesión docente, orientadas por principios neoliberalistas (Biscarra, Giaconi \& Assaél, 2015; Cornejo et al., 2015). Dichas regulaciones tienden a reorganizar la educación, tanto pública como privada, en virtud de principios gerencialistas que propugnan la evaluación estandarizada del desempeño docente, con incentivos y castigos asociados a los resultados (Cornejo et al., 2015; Fardella \& Sisto, 2015). De acuerdo con una serie de autores (Biscarra et al., 2015; Cornejo et al., 2015; Fardella \& Sisto, 2015), las nuevas reformas tienden a la desprofesionalización docente, siendo una manifestación de la desvalorización de la profesión.

Anteel escenario anterior,la respuestadesde el profesorado duranteelúltimo tiempo en Chile ha sido de rechazo, cuestión que se ha traducido en constantes movilizaciones, muchas veces impulsadas desde el propio magisterio (i.e. Colegio de Profesores de Chile). El debate sobre la profesión docente se ha instalado de esta forma en la opinión pública, siendo materia constante de discusión política. En este contexto resulta relevante conocer cuál es la visión que tienen los propios docentes o los estudiantes de pedagogía sobre la profesión docente y la figura del profesor.

Dentro de la lingüística cognitiva, una de las herramientas ampliamente utilizadas para indagar las conceptualizaciones de diferentes actores educativos sobre aspectos referidos a su profesión, es la metáfora conceptual. El valor de los estudios que adoptan dicho marco teórico radica en que exploran las creencias de los sujetos desde una dimensión compleja, fuertemente vinculada al contexto en el que estos se desenvuelven. De esta forma, este estudio intenta responder la pregunta de investigación: ¿qué conceptualizaciones metafóricas tienen los estudiantes de pedagogía sobre la figura del docente?

\section{Marco teórico}

El estudio sobre metáfora conceptual se inscribe dentro de la lingüística cognitiva, corriente que aúna una serie de teorías, enfoques, modelos, en definitiva, programas de investigación, que poseen como común denominador concebir el lenguaje como una capacidad integrada a la cognición humana general (Ibarretxe- Antuñano \& Valenzuela, 2012). Entre los fundamentos de la lingüística cognitiva se destaca, además, la adopción de un enfoque experiencialista, el cual señala que nuestraexperienciaincidefuertemente en la estructuración de nuestro pensamiento. 
La metáfora, en el ámbito de la lingüística cognitiva, se entiende como un recurso cognitivo que permite comprender un dominio de conocimiento más abstracto, llamado dominio meta, en relación con otro dominio más concreto, denominado dominio fuente, el cual se vincula a la experiencia de los sujetos. El conjunto de correspondencias entre elementos de ambos dominios constituye una metáfora conceptual. Uno de los presupuestos básicos de la teoría de la metáfora conceptual es concebir la metáfora como una parte fundamental de nuestro sistema conceptual ordinario, cuestión que se manifiesta en el hecho de que gran parte de nuestras acciones o de lo que decimos posee una base metafórica (Lakoff \& Johnson, 2012; Semino, Demjen, Hardie, Payne \& Rayson, 2018).

Dicho esto, se puede añadir como ejemplo la metáfora EL PROFESOR ES UNA BASE DE UNA CONSTRUCCIÓN, donde el dominio meta EL PROFESOR permite ser entendido en términos del dominio fuente, a saber, BASE DE UNA CONSTRUCCIÓN. A partir de esta asociación, se entienden frases como los profesores son la base de la sociedad, los profesores son los cimientos del futuro de nuestros estudiantes, sin profesores la sociedad se caería a pedazos, etc. Basándonos en este enfoque, entender los profesores como la base de una construcción implica no solo que expresemos por medio del lenguaje dicha asociación, sino también que nos comportemos y valoremos al profesorado de acuerdo con este concepto metafórico.

Saban (2006) y Black y Halliwell (2000) destacan el rol de la metáfora como un medio de reflexión profesional. Saban afirma que "By reflecting on personal thoughts, beliefs, and feelings, for example, metaphors provide a valid source for gaining insights into the teaching and learning from the perspectives of various school people" (2006, pp. 301-302). Enunciar metáforas a partir de la experiencia pedagógica puede ayudar a los docentes a comprender en qué medio se desenvuelven, tal como se ha reportado a través de grupos de discusión (Alarcón, Díaz, Vergara, Vásquez \& Torres, 2018). Además, la metáfora ha sido utilizada para fines investigativos como una herramienta de colección de datos y de análisis (Saban, 2006, p. 307). En este ámbito, uno de los campos más abordados ha sido el estudio de las creencias de diferentes actores del sistema educativo (Wan \& Low, 2015). Destacan las investigaciones sobre las creencias de docentes, tanto en formación (Saban, 2004; Saban, 2010; Thomas \& Beauchamp, 2011) como en ejercicio (Ben- Peretz, Mendelson \& Kron, 2003; Alger, 2009).

Uno de los métodos ampliamente utilizados para acceder a las creencias de los profesores ha sido la obtención metáforica (Low, 2015; Seung, Park \& Jung, 2015). Esta se constituye como un procedimiento donde un investigador motiva la producción 
de expresiones metafóricas en un sujeto, utilizando diferentes métodos (Low, 2015). En el estudio de las creencias docentes se han utilizado, por ejemplo: entrevistas (Thomas \& Beauchamp, 2011), dibujos (ver Ben-Peretz et al., 2003), encuestas (ver Saban, 2004; Alger, 2009), etc., o una combinación de estos métodos (Seung et al., 2015). Un procedimiento que destaca por su amplia utilización son las entradas escritas o written prompt (Saban, 2010; Seung et al., 2015; Alarcón et al., 2018), las cuales se establecen como frases incompletas (como a "teacher is like a...") que estimulan la generación de expresiones metafóricas (Seung et al., 2015). Dado que la metáfora conceptual es selectiva, es decir, que resalta ciertos elementos y aspectos del fenómeno que se intenta comprender (Saban, 2010; Lakoff \& Johnson, 2012; Semino et al., 2018), Yob plantea que mediante el empleo de una variedad de metáforas "a more comprehensive understanding of the subject can emerge" (2003, p. 299). Es decir, ninguna metáfora podrá reflejar por sí sola la complejidad de un fenómeno (como las creencias sobre la figura del docente), por lo que se requiere una multiplicidad de ellas para extraer una visión más o menos contundente acerca del mismo (Saban, 2006; Saban, 2010). En este sentido, la utilización de entradas escritas, dado que permiten su aplicación a una cantidad numerosa de sujetos (Seung et al., 2015), resultan apropiadas para tales fines.

A partir de este tipo de estudios, investigaciones como las de Saban (2004), Saban, Kocbeker y Saban (2007), Alger (2009), Saban (2010), dan cuenta de la organización de metáforas en categorías recurrentes. Una de las clasificaciones más referidas es la de Saban (2004), quien organiza las categorías de metáforas en dos perspectivas educacionales prominentes:

Metáforas centradas en el profesor, en la que la enseñanza se caracteriza por la transmisión de conocimientos. El profesor y el estudiante se describen respectivamente como: (1) transmisor de conocimientos y recipiente; (2) alfarero y materia prima; (3) reparador e individuo defectuoso; (4) figura de autoridad y sujeto dócil.

Metáforas centradas en el estudiante, que enfatizan la facilitación del aprendizaje y la participación activa del estudiante dentro del proceso de enseñanza- aprendizaje. El profesor y el estudiante se entienden como: (1) sujeto que nutre y organismo en desarrollo; animador y observador consciente; (3) andamio y constructor de conocimiento; (4) líder cooperativo y participante activo. Esta clasificación ha sido usada por diversos autores (Dincer, Yeşilyurt, Noels \& Vargas, 2019; Mellado, De la Montaña, Luengo \& Bermejo, 2016; solo por nombrar algunos) y fue usada como referencia para corroborar las categorías identificadas en el análisis de contenido de los datos. 


\section{Metodología}

El objetivo de la investigación fue analizar las conceptualizaciones metafóricas sobre la figura del profesor, en un grupo de estudiantes de pedagogía en inglés provenientes de diferentes universidades chilenas. En el estudio se empleó un enfoque cualitativo y se adoptó un diseño fenomenológico, ya que se busca comprender y caracterizar los fenómenos desde el punto de vista de los participantes (Creswell, 2007). Con ello, "se pretende reconocer las percepciones de las personas y el significado de un fenómeno 0 experiencia" (Hernández, Fernández \& Baptista, 2010, p. 515). Consecuentemente, el estudio se orienta a caracterizar los conceptos metafóricos que surgen desde el punto de vista de los participantes, estudiantes de pedagogía. Junto con esto, en lugar de utilizar un grupo numeroso de sujetos, la investigación cualitativa tiende a trabajar con menos participantes de lo que se hace en las investigaciones cuantitativas, dado que no tiene como finalidad generalizar de manera probabilística los resultados a poblaciones más amplias (Mackey \& Gass, 2005, p. 163; Hernández et al., 2010, p. 10).

\section{Participantes}

En el estudio participaron 163 estudiantes de pedagogía en inglés, provenientes de diferentes universidades chilenas. Entre los datos sociodemográficos más relevantes de los participantes se cuentan:

i. Sexo: la muestra la conforman principalmente mujeres (62,5\%). Un $27,2 \%$ corresponde a estudiantes hombres. En tanto, un 10,3\% de los participantes no respondió este ítem.

ii. Edad: la mayoría de los participantes tiene entre 21 y 23 años (51\%), seguido por quienes poseen entre 24 y 26 años (26 \%) y 27 a 29 años (9\%). Solo un $3 \%$ posee 30 años o más. Un $11 \%$ no respondió este ítem.

iii. Años de estudio: el $45 \%$ de los estudiantes cursaba su último año de universidad al momento de aplicado el instrumento, mientras que el 44 $\%$ se encontraba en su penúltimo año de estudio. El $11 \%$ de la muestra no respondió este ítem.

\section{Instrumentos de recolección de datos}

En el estudio se utilizó un cuestionario de obtención de metáforas (ver anexo), el cual incorpora entradas del tipo X es como Y, porque (ver Saban et al., 2007; Saban, 2010), para motivar la producción de expresiones metafóricas; donde $X$ corresponde al 
dominio fuente e $Y$, al dominio meta, mientras que la justificación de la metáfora otorgada se elabora en la respuesta mediante el conector "porque". Esta parte es fundamental para determinar los atributos que permiten categorizar la metáfora. El instrumento consta de dos partes. En la primera, se explica qué es la metáfora conceptual y se desarrollan ejemplos de metáforas bien construidas, es decir, donde hay dos dominios conceptuales y es posible identificar correspondencias entre ellos; todo ello con el fin de asegurar la mayor cantidad de respuestas válidas. Del mismo modo, se dan ejemplos donde no hay metáforas, sino descripciones literales de un concepto. En la segunda parte, el presente estudio aborda la pregunta: según su opinión, el profesor/la profesora es como... ¿Por qué?

A través de la firma de un consentimiento informado, los sujetos accedieron a responder de manera voluntaria el cuestionario, sin que esto formara parte de una actividad lectiva.

\section{Procedimiento de análisis}

Para el análisis de las expresiones metafóricas obtenidas mediante la aplicación del cuestionario se empleó un análisis de contenido semántico. En específico, se implementó el método propuesto por Saban et al. (2007) y Saban (2010) (aunque con algunas modificaciones), el cual consta de cinco fases: (a) preanálisis, (b) etiquetado, (c) clasificación, (d) categorización y (e) triangulación por investigadores.

a). Preanálisis: se definen las etapas mediante las cuales se analizarán los datos.

b). Etiquetado: se elabora una lista preliminar de las metáforas mediante la codificación del término o concepto proveído por cada participante para metaforizar la figura del profesor (e.g. MADRE, LUZ, JARDINER0, etc.). En caso de omisión de dicho término o de no haber respuesta, se excluían las expresiones de las siguientes etapas de análisis.

c). Clasificación: en un primer momento, se analizan las metáforas según tres aspectos, a saber: (i) dominio fuente, (ii) dominio meta y (iii) correspondencias. En un segundo momento, se eliminan las expresiones que, primero, no manifiestan una relación metafórica entre dos dominios específicos y, segundo, no presentan una justificación coherente que explique dicha relación. De esta forma, se obtienen rasgos prominentes para cada metáfora para continuar con la siguiente fase. 
d). Categorización: se analizan las metáforas según cómo es representada la figura del docente, del estudiante y el proceso de enseñanza y aprendizaje. De esta forma, se establece un listado de atributos que permite agrupar las metáforas según su similitud semántica. A cada conjunto se le otorga un rótulo representativo, estableciendo una serie de subcategorías. Luego, se realiza una revisión para reagrupar las metáforas en conjuntos más abarcables, estableciendo así categorías conceptuales. Finalmente, se organizan dichas categorías en macrocategorías, un nivel de abstracción superior, cuyos atributos se refieren, de forma general, a la labor pedagógica del docente 0 a su reconocimiento. Cabe señalar que no todas las metáforas pudieron ser agrupadas en categorías, pues para el establecimiento de las mismas debían presentarse, por lo menos, tres miembros con rasgos asimilables para cada conjunto. Las metáforas que no lograron incluirse en alguna categoría fueron etiquetadas como "problemáticas".

e). Triangulación por investigadores: se compara y somete a discusión la codificación y la categorización entre los cuatro investigadores a medida que se analizan los datos, con el propósito de obtener el mayor grado de consenso en el análisis. Asimismo, se toman decisiones acerca de las metáforas en que hubo disenso buscando una nueva categoría común o eliminándola.

\section{Resultados}

163 sujetos respondieron el cuestionario. Cuatro participantes omitieron su respuesta. De las respuestas analizadas, 12 se consideraron no metafóricas, por ende, el número total de metáforas suma 151.

Asimismo, a partir del proceso de triangulación se excluyeron de la categorización 17 metáforas, dado que no se logró adscribirlas a alguna categoría, las cuales fueron catalogadas como "metáforas problemáticas", esto es, aquellas en las que no hubo consenso para adscribirlas a una metáfora determinada. Por ejemplo, un participante empleó la metáfora UNA CAJA DE PANDORA, argumentando que cada profesor tiene su propia manera y estilo de enseñanza (...) hay algunos que tienen mucha pacienciay tolerancia por sus alumnos y semejantes, mientras otros se sienten tristes y cansados por su profesión (...). Objetivamente bablando, un profesor para mí significa alguien realmente comprometido con lo que piensa, con lo que dice y lo que bace. En esta respuesta se metaforiza al profesorado, más que al concepto de profesor(a) en singular. Además, la última parte de la respuesta, que repara en el compromiso del 
docente, no se relaciona claramente con el dominio fuente CAJA DE PANDORA. En consecuencia, a pesar de ser metafórica, la respuesta se consideró como "problemática".

De esta forma, el número total de metáforas categorizadas es de 130. En la Tabla 1 se expone el detalle de los datos anteriores.

Tabla 1. Detalle de los datos recopilados mediante el cuestionario de obtención metafórica

\begin{tabular}{ccc}
\hline Encuestas & Número & $\begin{array}{c}\text { Distribución } \\
\text { porcentual (\%) }\end{array}$ \\
\hline Encuestas aplicadas & 163 & 100 \\
\hline $\begin{array}{c}\text { Encuestas sin información en la entrada } \\
\text { referida a la figura del docente }\end{array}$ & 4 & 2.5 \\
\hline $\begin{array}{c}\text { Respuestas consideradas no metafóricas } \\
\text { Metáforas problemáticas }\end{array}$ & 12 & 7.4 \\
\hline Metáforas categorizadas & 17 & 10.4 \\
\hline
\end{tabular}

A partir del análisis de contenido realizado se establecieron ocho categorías conceptuales, agrupadas en tres macrocategorías (o categorías conceptuales mayores), a saber, metáforas centradas en el profesor, metáforas centradas en el estudiante y metáforas centradas en el reconocimiento social del profesor. Las dos primeras, ya identificadas por Saban (2004), se restringen al proceso de enseñanza y aprendizaje, destacando la figura del docente 0 estudiante, respectivamente. Y la terceramacrocategoría destaca aspectos que no se restringen al proceso pedagógico dentro del aula, sino a la valoración de la labor del profesor, en virtud de su repercusión social.

Producto del proceso de triangulación por investigador, en la categorización se establecieron atributos de las metáforas no solo reparando en el conocimiento general que se tiene sobre el dominio fuente propuesto por el participante (HORMIGA,GUÍA,AGUA, entre otros), sino que también se consideraron los rasgos sobre la figura del profesor que son destacados en la fundamentación de la metáfora. Como se sostiene en la literatura en la materia (Kövecses, 2002; Lakoff, 1993; Lakoff \& Johnson, 2012), las metáforas hacen que un concepto se torne más comprensible, focalizándose en ciertos aspectos del dominio meta. Es decir, las correspondencias enfatizan determinadas características del concepto 
metaforizado y ocultan otras. De esta manera, una misma metáfora o dos de ellas, que incluyan un dominio fuente similar (por ejemplo, LUZ y LÁMPARA) pueden inscribirse en categorías diferentes, en la medida en que las fundamentaciones entregadas por los sujetos participantes (también de índole metafórica), expresen dos visiones sobre el papel del profesor, disímiles entre sí.

En la Tabla 2 se exponen los resultados de la categorización realizada, la cual integra la distribución porcentual de cada categoría, en relación con el total de metáforas categorizadas (i.e. 130).

Tabla 2. Clasificación de metáforas referidas a la figura del profesor

\begin{tabular}{cccc}
\hline $\begin{array}{c}\text { Macrocategoría } \\
\text { (distribución porcentual) }\end{array}$ & $\begin{array}{c}\text { Categoría (número } \\
\text { de miembros) }\end{array}$ & $\begin{array}{c}\text { Distribución } \\
\text { porcentual (\%) }\end{array}$ & Ejemplos de metáforas \\
\hline $\begin{array}{c}\text { Metáforas centradas en el } \\
\text { estudiante (65,38\%) }\end{array}$ & Animador & 4.62 & $\begin{array}{c}\text { Artista, humorista, mago, } \\
\text { cantante pop, etc. }\end{array}$ \\
\hline & Cuidador & 29.23 & Jardinero, madre, padre, etc. \\
\cline { 2 - 4 } & Mediador & 31.54 & $\begin{array}{c}\text { Guía, luz, director técnico, } \\
\text { mapa. }\end{array}$ \\
\hline $\begin{array}{c}\text { Metáforas centradas en el } \\
\text { profesor }\end{array}$ & $\begin{array}{c}\text { Fuente de } \\
\text { conocimientos (9) }\end{array}$ & 6.92 & $\begin{array}{c}\text { Enciclopedia, libro, lámpara, } \\
\text { etc. }\end{array}$ \\
\hline $\begin{array}{c}\text { Metáforas } \\
\text { Moldeador } \\
(10)\end{array}$ & $\begin{array}{c}\text { Guerrero } \\
(12)\end{array}$ & 7.69 & $\begin{array}{c}\text { Escultor, Carpintero, maestro } \\
\text { de construcción, etc. }\end{array}$ \\
\hline $\begin{array}{c}\text { Recurso } \\
\text { vital (7) }\end{array}$ & 9.23 & 5.38 & $\begin{array}{c}\text { Guerrero, } \\
\text { Social del profesor (20\%) }\end{array}$ \\
\hline
\end{tabular}

Dentro de las macrocategorías establecidas, las metáforas centradas en el estudiante fueron preponderantes, obteniendo un $65,38 \%$ del total de metáforas categorizadas. Dentro de esta, destaca la categoría mediador, la cual abarca la mayor 
cantidad de metáforas (31,54\%). Paralelamente, la macrocategoría con menor porcentaje de miembros fue metáforas centradas en el profesor (14,62 \%), la cual incluye las categorías fuente de conocimientos y moldeador, con un 6,92 \% y 7,69\% de distribución porcentual respectivamente. La categoría que aglutina la menor cantidad de metáforas categorizadas fue animador con un 4,62 \%. A continuación, se detalla cada una de las categorías establecidas, posterior al análisis de contenido realizado.

\section{Metáforas centradas en el estudiante}

\section{Animador}

En esta categoría, el profesor se entiende como un agente que debe lograr captar la atención de los estudiantes durante su intervención y motivarlos e involucrarlos en el proceso pedagógico durante el desarrollo de la clase; por ejemplo, los participantes sostienen que el profesor siempre está buscando nuevas rutinas para 'entretener' a los alumnos (EL PROFESOR ES UN HUMORISTA) o que constantemente está motivando y creando diversas actividades y así no aburrir a los educandos (EL PROFESOR ES UN ARTISTA). El objetivo es generar un aprendizaje significativo en los estudiantes; en palabras de uno de los participantes: [que la clase] todos la disfruten y la recuerden (EL PROFESOR ES UN ACTOR DE TEATRO). El educando, de esta forma, se involucra con el proceso de enseñanza y aprendizaje, pero no necesariamente participando activamente dentro del mismo. Varias de las metáforas definen al grupo curso como una audiencia, la cual si bien mantiene la atención, parece tener menor grado de incidencia en el proceso (EL PROFESOR ES UN CANTANTE POP, EL PROFESOR ES UN ACTOR DE TEATRO y EL PROFESOR ES UN HUMORISTA).

\section{Cuidador}

De acuerdo con esta categoría, el docente se establece como la figura que se encarga del desarrollo personal del estudiante. La labor pedagógica, de esta forma, se entiende en términos amplios; es decir, no se restringe a la mera transmisión de conocimientos, sino que implica también inculcar valores, entregar apoyo emocional, brindar protección, realizar, en definitiva, una labor de orientación.

Desde esta categoría, se extraen dos perspectivas; por un lado, las metáforas que refieren al profesor como un ente protector que debe asegurar un ambiente propicio para el crecimiento personal del estudiante, brindando además los cuidados necesarios para que este se desarrolle como persona, los cuales se asocian a oficios como la jardinería, por ejemplo: [El profesor] se preocupa de que sus flores, que en este caso son los 
alumnos, crezcan y se desarrollen de la mejor manera posible como personas, proveyéndolos de agua y luz, que son el conocimiento y los valores (EL PROFESOR ES UN JARDINER0). Muchas veces se hace hincapié en los resultados de la labor docente; en este sentido, la metáfora EL PROFESOR ES UN AGRICULTOR refiere que los profesores proveen las herramientas necesarias para que la enseñanza rinda frutos. Por otro lado, una segunda perspectiva ofrece una imagen más humanizada del vínculo entre docente y estudiante, reparando con más ahínco en labor de orientación personal que media entre ambos. Así, por ejemplo, el profesor se encarga de dar consejos adecuados según la edady la necesidad de cada uno [estudiante] (EL PROFESOR ES UNA MADRE) o debe no solo enseñar contenidos, sino también valores, [a los estudiantes debe] cuidarlos, escucharlos, comprenderlos y aconsejarlos (EL PROFESOR ES UN PADRE).

\section{Mediador}

El proceso de enseñanza y aprendizaje exige la involucración activa del estudiante, donde la labor del docente consiste en orientar la participación de este. El profesor es quien media en la consecución de aprendizajes. Ahora bien, en la presente categoría coexisten metáforas que implican un grado diferente de participación, tanto del profesor como del estudiante, aunque todas estas se centran en lograr una experiencia de aprendizaje en este último. Dentro de la presente categoría tenemos un primer conjunto de metáforas que refieren al docente como quien debe dirigir a los estudiantes para que logren como grupo metas comunes de aprendizaje. Por ejemplo, en la metáfora EL PROFESOR ES UN ENTRENADOR DE FÚTBOL se plantea que el docente tiene un grupo de personas con distintas características y logra hacer que trabajen en conjunto para lograr un objetivo más grande. Asimismo, abundan las metáforas que entienden el proceso de enseñanza y aprendizaje como un viaje, por ejemplo en la metáfora EL PROFESOR ES UN FARO se señala que:

[El profesor] guía y alumbra tu camino. Por muy lejos que estés de tierra, con su luz te dice que puedes llegar y te muestra siempre el mejor puerto donde encallar. El profesor o profesora siempre ilumina tu camino y te cobija en tierra firme. Al alumno le muestra el camino y lo acompaña y al final lo felicita por construir su propio destino.

De acuerdo con el ejemplo, se acentúa la noción de proceso, inherente a la labor pedagógica del docente, que en este caso está orientada hacialos resultados de aprendizajes. Finalmente, un último grupo de metáforas otorga un mayor protagonismo al estudiante. El profesor aquí ofrece las herramientas necesarias para que el educando pueda incidir de forma autónoma en su propio aprendizaje. Ejemplo de esto es la metáfora EL PROFESOR 
ES UNA CAJA DE HERRAMIENTAS, la cual presenta la siguiente justificación [el profesor] te da lo que necesitas para realizar alguna obra, (...) te entrega los conocimientos o berramientas para ejecutar alguna acción, pero el responsable de los resultados eres tú [el estudiante], ya que de ti depende el uso que le des a esas berramientas. Con todo, se evidencia que, a diferencia de la categoría cuidador, el mediador asume una tarea inherentemente pedagógica, que no se concentra en la relación afectiva que posee el estudiante, sino en la labor pedagógica propiamente tal.

\section{Metáforas centradas en el profesor}

\section{Fuente de conocimientos}

La figura del docente se define a partir de los conocimientos que este ostenta y, en especial, por su capacidad para poder transmitirlos a los estudiantes. Estos últimos poseen un papel pasivo dentro del proceso de enseñanza, funcionando como un receptáculo de saberes provistos por el docente. Así, por ejemplo, la metáfora EL PROFESOR ES UNA FUENTE presenta la siguiente justificación: de la fuente emana el agua que es el conocimiento que nos permite saciar nuestra sed de sabiduría. Se valora, por un lado, la cantidad de conocimientos que posee el docente, los cuales muchas veces trascienden lo restrictivo a su disciplina: el profesor no solo debe poseer los conocimientos relacionados con su materia, sino que debe ser capaz de entregar conocimientos asociados también a las demás asignaturas (EL PROFESOR ES UNA ENCICLOPEDIA). Por otro lado, se apela a la calidad de los saberes de los profesores y a la disposición de estos: los docentes poseen grandes conocimientos, (...) se puede recurrir a ellos cada vez que necesitemos información (EL PROFESOR ES UN LIBRO ABIERTO).

\section{Moldeador}

Esta categoría destaca la concepción de la enseñanza como una labor de formación, que involucra al estudiante no solo en términos de sus conocimientos, sino también de sus valores, actitudes, habilidades, etc. El profesor es el sujeto cualificado para realizar la tarea formativa, mientras que el estudiante posee un papel pasivo dentro del proceso. Ahora bien, algunas metáforas destacan las características únicas que tiene cada estudiante, así como las habilidades o potencialidades que deben ser desarrolladas; por ejemplo, en la metáfora EL PROFESOR ES UN ESCULTOR se señala que: [el docente] debe sacar lo mejor de la materia prima que tiene en frente, es decir, los alumnos. Con dedicación, suavidad, con entereza, tal cual lo bace un escultor con sus esculturas. 
Dentro del conjunto, el profesor es quien adquiere el protagonismo; su misión es formar estudiantes útiles para la sociedad; es decir, que operen con eficacia dentro del marco social, contribuyendo de alguna forma a la misma, gracias a los aprendizajes obtenidos. Así, por ejemplo, en la metáfora EL PROFESOR ES UN CARPINTERO se señala que a los alumnos [el profesor] los toma como madera cuadrada y poco a poco va dándoles forma hasta que los finaliza. Es un trabajo sacrificado y detallado que al final tiene sus frutos para que sirvan en la sociedad. Como se evidencia, la profesión docente se conceptualiza vinculándola a otros rubros como la carpintería, la artesanía 0 al campo artístico (la escultura, por ejemplo). Se acentúa, asimismo, la dedicación que demanda el ejercicio de la pedagogía.

\section{Metáforas centradas en el reconocimiento social del profesor}

\section{Guerrero}

En esta categoría se ubican las metáforas que ofrecen una imagen positiva del profesor, dado que este debe luchar para ejercer su profesión y educar a sus estudiantes. En este sentido, los participantes refieren aspectos que pueden obstaculizar u oponerse a la labor educativa del docente, los cuales pueden restringirse al proceso de enseñanza y aprendizaje 0 vincularse a las condiciones laborales o sociales de la profesión. Así, por ejemplo, se señala que [el docente] tiene que luchar contra la adversidad y complicaciones que se pueden dar tanto dentro como fuera de la sala de clases (EL PROFESOR ES UN GUERRER0). Asimismo, un participante señala que:

hay que luchar para sacarse un estigma, hay que luchar para que los niños aprendan (estos niños quienes se les ha enseñado toda la vida que estudiar/saber no es divertido). Deben también luchar contra los alumnos, apoderados, sistema... en fin... la eterna lucha.

Como se evidencia, son muchos los enemigos que se oponen al ejercicio docente. Otras veces se plantea que el profesor se inserta en un ambiente desfavorable para desempeñarse como profesor.

\section{Pilar}

En términos generales, esta categoría señala que la labor docente sustenta el futuro desenvolvimiento del estudiante dentro de la sociedad. En este sentido, se acentúa el efecto positivo (o negativo) de la labor pedagógica sobre el futuro del educado, entendiéndolo como ciudadano o miembro de la sociedad. Así también, frecuentemente se apunta al docente como aquel que otorga sustento a la sociedad en 
general; es decir, quien se encarga de educar a las personas desde sus inicios, siendo la base de las restantes profesiones u ocupaciones: los profesores son los que cimientan las bases de la sociedad. Son los encargados de formar a todos, tanto en los ámbitos académicos y sociales. Sin profesores la sociedad se caería a pedazos (EL PROFESOR ES CEMENTO).

\section{Recurso vital}

Los docentes se conciben como fundamentales para la vida de los estudiantes. Estos últimos deben desarrollarse como personas y, para esto, resulta indispensable la acción docente, así como lo son el agua, el aire o la luz para otros organismos. En este sentido, un participante señala: la luz es un factor muy importante para que crezcan las plantas, al igual que los profesores son un factor muy importantepara el desarrollo del estudiante (EL PROFESOR ES UNA LUZ).

\section{Discusión}

En América Latina, durante el último tiempo, se han implementado consistentemente reformas a la labor docente apoyadas en una visión tecnificada de la misma (Tenti, 2005; Ávalos, Cavada, Pardo \& Sotomayor, 2010). En el caso chileno, la última década ha estado marcada por el avance de reformas neoliberales, las cuales establecen nuevas funciones a los docentes (Biscarra et al., 2015; Cornejo et al., 2015). Este contexto permite explicar la categoría de GUERRER0, establecida mediante el análisis de contenido realizado. Esta categoría da cuenta de las condiciones adversas y demandantes en las que se inserta el profesorado. En este marco, los docentes deben enfrentarse arduamente a las complicaciones producidas por su ejercicio profesional, entre las que se cuentan aspectos relacionados con el proceso de enseñanza y aprendizaje u otros asociados a la desvalorización social o las malas condiciones laborales que les afectan.

El que no se hayan podido establecer categorías que, prominentemente, definan la figura del profesor de forma negativa, evidencia que este grupo de estudiantes, si bien son conscientes de las dificultades de su futura profesión o del contexto hostil en la que esta se inserta (constatable, por ejemplo, en la categoría guerrero), parecen estar comprometidos y satisfechos con la labor educativa que desempeña el profesor. Estos resultados se condicen con evidencias sobre la visión que poseen profesores chilenos en ejercicio, reportadas por Ávalos y Sotomayor (2012), las cuales señalan, después de estudiar una muestra representativa de la población docente en Chile, que los docentes manifiestan una identidad profesional positiva y expresan estar motivados 
y comprometidos con sus tareas, a pesar de la tensión que suponen los constantes cambios en el sistema educativo y la desvalorización de su profesión.

Es así como las categorías guerrero, pilar, recurso vital dan cuenta de la valoración positiva hacia la profesión. De acuerdo con estas, el docente resulta indispensable, dado que este se encarga de educar a la población, a pesar de las dificultades que debe enfrentar para ejercer su ejercicio profesional. En este aspecto, destacan metáforas como EL PROFESOR ES UN SUPERHÉROE, adscritas a la categoría guerrero, EL PROFESOR ES UNA MADRE y EL PROFESOR ES UN PADRE, ambas pertenecientes a la categoría mediador; esto, en la medida en la que implican una visión del docente como modelo. En este sentido, uno de los participantes refiere que: un profesor es el primer ejemplo (...) de modales, educación y conocimiento que un niño tiene (EL PROFESOR ES UN SUPERHÉROE).

Estos resultados son coincidentes con los del estudio realizado en el marco del proyecto FONDECYT N. ${ }^{\circ} 1150889$ (Díaz, Alarcón, Tagle, Quintana, Ramos, 2018), en el cual se abordan las actitudes hacia la profesión docente mediante una escala tipo Likert en el mismo grupo de participantes que los del presente trabajo. El análisis muestra que los ítems que obtienen la percepción más favorable hacia la profesión docente corresponden a: 7) "Los profesores deben estar orgullosos de su profesión, independientemente de los sentimientos que otros tengan hacia ella" (77,6\% Totalmente de acuerdo); 14) "La enseñanza es una profesión desafiante" (85,6 \% Totalmente de acuerdo); 19) "La enseñanza es una profesión respetable" (74,6 \% Totalmente de acuerdo). Por otro lado, entre los ítems pertinentes al presente estudio que presentan la percepción más desfavorable hacia el ejercicio de la profesión docente se encuentra: 18) "La profesión docente permite tiempo libre a los profesores" (47,1\% Totalmente en desacuerdo y 32,2\% En desacuerdo). En otras palabras, los resultados del análisis de metáforas y de la escala de actitudes evidencian que los futuros profesores están conscientes de las dificultades del ejercicio profesional y aun así presentan una actitud positiva hacia la profesión, que consideran desafiante. Las condiciones de desempeño requieren de la fuerza de un guerrero y de grandes poderes, como los de un superhéroe. Por ello, no es de extrañar que los sujetos manifiesten estar orgullosos de la profesión que eligieron.

En definitiva, si bien la labor docente se considera demandante, los estudiantes de pedagogía están dispuestos a ejercerla, dada su importancia social y la influencia que ejerce el docente en la vida de sus alumnos. 
Así también, los estudiantes definen al profesor en virtud de la consecución de aprendizajes de sus educandos. Destaca, dentro del conjunto de metáforas, la visión del docente como mediador: Los futuros profesores conciben que se debe guiar al estudiante para posibilitar su aprendizaje, por ende, este último debe poseer una participación activa dentro del proceso educativo. Más aún, es tarea del docente otorgar las herramientas para que el educando alcance su aprendizaje. De esta forma, se hace manifiesta una perspectiva constructivista del proceso pedagógico.

Se debe destacar, además, que como conjunto de categorías, las metáforas centradas en el profesor resultaron ser las menos preponderantes. Los participantes conciben en menor medida al docente, por ejemplo, como transmisor de conocimientos, visión que puede ser atribuida a una perspectiva más tradicional del aprendizaje, en comparación con otras categorías que lo refieren, por ejemplo, como un mediador. En síntesis, la conceptualización del docente no se enfoca netamente en el desempeño de este, sino en su papel dentro de la dinámica de aprendizaje, en vinculación a la consecución de aprendizajes en sus educandos. Esto no solo implica la adquisición de conocimientos, sino también la inculcación de valores o el desarrollo emocional de estos últimos.

Estos resultados contrastan con los de estudios que han utilizado la misma metodología para el análisis de la metáfora en muestras similares de participantes (estudiantes de pedagogía en etapa de práctica profesional), donde predominan categorías relativas a una evaluación negativa de la profesión docente (Alarcón et al., 2018), ya sea porque los sujetos asumen que la profesión está desvalorizada socialmente: EL PROFESOR ES UN INSECTO, EL PROFESOR ES UN TESORO SIN VALOR, o porque destacan las malas condiciones de desempeño: EL PROFESOR ES UNA NIÑERA, EL PROFESOR ES UNA MÁQUINA, etc. Estas diferencias pueden estar motivadas por las experiencias de los sujetos, ya sea las adquiridas como estudiantes de educación primaria y secundaria 0 como alumnos de cursos finales de pedagogía que ya han visitado centros educacionales, como se pudo constatar a través de los grupos de discusión en los estudios previos. No se puede desconocer el contexto social; sin embargo, las demandas y movilizaciones estudiantiles y del profesorado vienen ocurriendo regularmente en Chile, por lo que no parecen ser un factor determinante en la visión más positiva de un grupo por sobre otro.

\section{Conclusiones}

Los futuros profesores que participaron en el estudio perciben la profesión docente como desafiante 0 demandante. No obstante, su actitud hacia esta es prominentemente positiva; es decir, están dispuestos a ejercerla a pesar de las futuras 
dificultades que involucra su ejercicio. Asimismo, los participantes poseen una visión del proceso pedagógico centrado en el educando, donde resulta contingente la visión de que el estudiante debe "descubrir" su propio aprendizaje, por ende, el docente debe mediar para que se produzca la consecución del mismo.

Resulta importante realizar un seguimiento longitudinal de las expresiones metafóricas y actitudes hacia la profesión docente durante el proceso formativo de un profesor en las universidades, de tal forma que se diagnostique inicialmente y se corrobore al final de la formación, si esta efectivamente tiene algún impacto en el tipo de actitud y metáfora que construye el futuro profesor. De esta manera, la formación recibida en la universidad puede profundizar en los procesos reflexivos de los futuros profesores, particularmente en aquellos que aporten en el desarrollo de estrategias y herramientas que los ayuden a enfrentar la complejidad de la enseñanza y el aprendizaje, y los escenarios contextuales donde ambos procesos se llevan a cabo.

Entre las debilidades del presente estudio, conviene mencionar que para la obtención de metáforas se utilizó solo el cuestionario. La literatura reciente, sin embargo, recomienda la aplicación de más de un método, pues así es posible triangular los datos obtenidos (Seung et al., 2015; Wan \& Low, 2015). Ahora bien, dicha sugerencia resulta también dificultosa, si se busca indagar en las creencias o conceptualizaciones metafóricas de una muestra numerosa de sujetos (Seung et al., 2015). No obstante, se espera realizar esfuerzos para diversificar los métodos de recolección de datos en las próximas investigaciones. De esta forma, se puede obtener mayor información acerca de las conceptualizaciones de los futuros profesores hacia la profesión, y también se pueden integrar aspectos como la motivación hacia la misma, por ejemplo.

Finalmente, la variabilidad de las metáforas y fundamentaciones, sumada a la extensión variable de las respuestas obtenidas por medio de las entradas escritas, pueden contribuir a una mayor discordancia en las categorizaciones realizadas por los investigadores. Conviene, de esta forma, establecer métodos que permitan controlar la calidad de las respuestas, por ejemplo, realizando un acompañamiento durante la aplicación del instrumento de investigación (Low, 2015), para así aclarar dudas o verificar que este se complete de manera correcta. De esta forma, se minimizaría la cantidad de respuestas no metafóricas $(7,4 \%$, en el caso de este estudio) o poco informativas, aunque también debe resguardarse en aquello que no se produzca algún tipo de sesgo por influencia del investigador. 


\section{Anexo}

FOLIO N. ${ }^{0}$

\section{CUESTIONARIO}

Estimado/a participante:

En el contexto de una investigación sobre conocimiento profesional docente solicitamos a usted completar la presente encuesta de la manera más precisa posible. De antemano agradecemos su disposición a colaborarnos. Su participación es voluntaria y la información aportada por usted será completamente anónima.

\section{Metáforas}

Antes de comenzar, revise cuidadosamente los siguientes ejemplos, donde se define y explica la metáfora:

\section{¿Qué es una metáfora?}

La metáfora es un concepto mediante el cual pensamos y hablamos sobre un ámbito de la realidad en términos de otro. Por ello, las metáforas pueden entenderse como un conjunto de relaciones o correspondencias entre esos dos ámbitos de la realidad involucrados.

En la tabla se muestran ejemplos correctos e incorrectos de metáforas. Observe que el nombre de la metáfora (en mayúscula) se enuncia como una comparación entre dos ámbitos de la realidad y luego se justifican las relaciones entre ambos.

\begin{tabular}{|c|c|}
\hline Ejemplos correctos de metáfora $\sqrt{ }$ & $\begin{array}{c}\text { Ejemplos incorrectos de metáfora } \\
\mathrm{X}\end{array}$ \\
\hline $\begin{array}{l}\text { A). EL MATRIMONIO ES COMO UN VIAJE. } \sqrt{ } \\
\text { ¿Por qué? porque las personas son como los viajeros } \\
\text { que se embarcan en el matrimonio. También cuando } \\
\text { hay problemas se puede decir que ya no se avanza o } \\
\text { que el camino ha estado lleno de obstáculos. Además, } \\
\text { cuando se piensa en la separación, se puede decidir } \\
\text { que es mejor que cada uno tome su propio camino. } \sqrt{ } \\
\text { B). EL MATRIMONIO ES COMO UNA CARGA. } \sqrt{ } \\
\text { ¿Por qué? porque cuando el matrimonio se hace } \\
\text { complicado, se vuelve una carga que no se puede } \\
\text { soportar. Por lo mismo, las personas se sienten } \\
\text { aliviadas cuando se deja de llevar sobre los hombros } \\
\text { ese peso que han arrastrado por años, etc. } \sqrt{ }\end{array}$ & $\begin{array}{l}\text { C). C) EL MATRIMONIO ES COMO UN } \\
\text { VIAJE. } \\
\text { ¿Por qué? Porque ambos tienen } \\
\text { duración y son realizados por } \\
\text { personas. X } \\
\text { D). D) EL MATRIMONIO ES COMO UNA } \\
\text { MOTIVACIÓN. X } \\
\text { ¿Por qué? porque el matrimonio da } \\
\text { una razón para vivir. X }\end{array}$ \\
\hline
\end{tabular}


RECUERDE QUE: Para que se produzca la metáfora, tiene que haber relaciones entre los elementos de dos ámbitos de la realidad.

Así, en el ejemplo correcto (A), las personas son como los viajeros, los problemas del matrimonio son obstáculos en el camino, etc.

En el ejemplo incorrecto $(\mathrm{C})$, decir solamente que los matrimonios son como viajes porque tienen duración es demasiado general, pues se dejan de lado relaciones más específicas de esa metáfora. El ejemplo (D) es incorrecto, porque la motivación es una idea que describe al matrimonio. No es un concepto que pertenezca a otro dominio de la realidad.

Los ejemplos correctos (A y B) nos muestran, además, que para un mismo concepto (el matrimonio) puede haber más de una metáfora, cada una con sus relaciones específicas.

Observe que una respuesta como "el profesor/a es como el responsable de la educación de los niños" no es metafórica, sino que es una descripción literal del rol docente.

\section{Después de haber revisado y leído los ejemplos, complete el siguiente enunciado y justifique su respuesta.}

Según su opinión, el profesor/la profesora es como... ¿Por qué?

\section{Referencias}

Alarcón, P.; Díaz, C., Vergara, J., Vásquez, V. \& Torres, C. (2018). Análisis de metáforas conceptuales sobre la imagen social del profesorado en estudiantes de pedagogía. Onomázein, 40, 1-27. https://doi.org/10.7764/onomazein.40.01

Alger, Ch. (2009). Secondary teachers' conceptual metaphors of teaching and learning: Changes over the career span. Teaching and Teacher Education, 25(5), 743-751. https://doi. org/10.1016/j.tate.2008.10.004

Amsterdam: John Benjamins. https://doi.org/10.1075/milcc.3.02seu

Ávalos, B., Cavada, P., Pardo, M. \& Sotomayor, C. (2010). La profesión docente: temas y discusiones en la literatura internacional. Estudios Pedagógicos, 35(1), 235-263. https://doi.org/10.4067/S0718-07052010000100013 
Ávalos, B. \& Sotomayor, C. (2012). Cómo ven su identidad los docentes chilenos. Revista Perspectiva Educacional, $\quad 51(1), \quad 57-84 . \quad$ https://doi.org/10.4151/07189729Vol.51-Iss.1-Art.74

Ben-Peretz, M., Mendelson, N. \& Kron, F. (2003). How teachers in different educational context view their roles. Teaching and Teacher Education, 19(2), 277-290. https://doi.org/10.1016/ S0742-051X(02)00100-2

Biscarra, C., Giaconi, C. \& Assaél, J. (2015). El docente en la legislación educacional chilena, Psicoperspectivas, 14(3), 80-92. Doi: 10.5027/PSICOPERSPECTIVASVOL14-ISSUE3-FULLTEXT-600 https://doi.org/10.5027/psicoperspectivas-Vol14-Issue3fulltext-600

Black, A. L., \& Halliwell, G. (2000). Accessing practical knowledge: How? Why? Teaching and Teacher Education, 16(1), 103-115. https://doi.org/10.1016/S0742-051X(99)00045-1

Cornejo, R., Albornoz, N. Castañeda, L., Palacios, D. Etcheberrigaray, G., Fernández, R. \& Lagos, J. I. (2015). Las prescripciones del trabajo docente en el nuevo marco regulatorio de políticas educativas en Chile. Psicoperspectivas, 14(2), 72-83. https://doi.org/10.5027/ psicoperspectivas-Vol14-Issue2-fulltext-580

Creswell, J. (2007). Qualitative Inquiry and Research Design. Choosing among Five Approaches. Londres: Sage.

Díaz, C., Alarcón, P., Tagle, T., Quintana, M. \& Ramos, L. (2018). Las dimensiones cognitivas, afectivas y sociales del proceso de planificación de aula y su relación con los desempeños pedagógicos en estudiantes de práctica profesional y profesores nóveles de pedagogía en inglés. Proyecto Fondecyt n. ${ }^{0} 1150889$. Concepción, Chile: Universidad de Concepción.

Dincer, A., Yeşilyurt, S., Noels, K. A. \& Vargas Lascano, D. I. (2019). Self- determination and classroom engagement of EFL Learners: A mixed-methods study of the self-system model of motivational development. Sage Open, 9(2). https://doi.org/10.1177/2158244019853913

Discurso, subjetividad y resistencia. Psicologia \& Soceidade, 27(1), 68-79. Recuperado de http://www. redalyc.org/pdf/3093/309338439008.pdfhttps://doi.org/10.150/1807-03102015v27n1p068

Fardella, C. \& Sisto, V. (2015). Nuevas regulaciones del trabajo docente en Chile.

Hernández, C., Fernández, C. \& Baptista, P. (2006). Metodología de la investigación (4.a ed.). México D. F.: McGraw-Hill.

Ibarretxe-Antuñano, I. \& Valenzuela, J. (2012). Lingüística cognitiva: origen, principios y tendencias. En I. Ibarretxe-Antuñano \& J. Valenzuela (coord.) Lingüística cognitiva (pp. 12-38). Barcelona: Anthropos. 
Kövecses, Z. (2002). Metaphor: A Practical Introduction. Oxford/Nueva York: Oxford University Press.

Lakoff, G. (1993). The contemporary theory of metaphor. En. A. Ortony (ed.), Metaphor and thought (pp. 202-251). Cambridge: Cambridge University Press. https://doi.org/10.1017/ CB09781139173865.013

Lakoff, G. \& Johnson, M. (2012). Metáforas de la vida cotidiana (9. ed.). Madrid: Cátedra.

Low, G. (2015). A practical validation model for researching elicited metaphor. In W.

Mackey, A. \& Gass, S. (2005). Second language research. Methodology and design. Mahwah, NJ: Lawrence Erlbaum.

Mellado, L., Montaña de la, J., Luengo, M. \& Bermejo, M. (2016). Evolución de los modelos docentes de futuros profesores de economía de secundaria, a través de la metáfora. Didáctica de las ciencias experimentales y sociales, 30, 23-44. https://doi.org/10.7203/ dces. 30.7230

Saban, A. (2004). Prospective classroom teachers' metaphorical images of selves and comparing them to those they have of their elementary and cooperating teachers. International Journal of Educational Development, 24(6), 617-635. https://doi.org/10.1016/j. ijedudev.2004.03.003

Saban, A. (2006). Functions of metaphor in teaching and teacher education: A review essay. Teaching Education, 17(4), 299-315. https://doi.org/10.1080/10476210601017386

Saban, A. (2010). Prospective teachers' metaphorical conceptualizations of learner, Teaching and Teacher Education, 26(2), 290-305. https://doi.org/10.1016/j.tate.2009.03.017

Saban, A., Kocbeker, B. N. \& Saban, A. (2007). Prospective teachers' conceptions of teaching and learning revealed through metaphor analysis, Learning and Instruction, 17(2), 123-139. https://doi.org/10.1016/j.learninstruc.2007.01.003

Seung, E., Park, S. \& Jung, J. (2015). Methodological approaches and strategies for elicited metaphor-based research. A critical review. In W. Wan \& G. Low (eds.), Elicited metaphor analysis in educational discourse (pp. 39-64).

Semino, E., Demjen, Z., Hardie, a., payne, s. \& rayson, p. (2018). metaphor, cancer and the end of life: A corpus-based study. London: Routledge. https://doi.org/10.4324/9781315629834

Tenti, E. (2005). La condición docente. Análisis comparado de la Argentina, Brasil, Perú y Uruguay. Buenos Aires, Argentina: Siglo Veintiuno. 
Thomas, L. \& Beauchamp, C. (2011). Understanding new teachers' professional identities through metaphor. Teaching and Teacher Education, 27, 762-769. https://doi. org/10.1016/j.tate.2010.12.007

Wan, W. \& Low, G. (2015). Introduction. In W. Wan \& G. Low (Eds.). Elicited Metaphor Analysis in Educational Discourse (pp. 1-12). Amsterdam: John Benjamins. https://doi.org/10.1075/ milcc.3.001int

Wan \& G. Low (eds.), Elicited metaphor analysis in educational discourse (pp. 15-37). Amsterdam: John Benjamins. https://doi.org/10.1075/milcc.3.01low

Yob, I. M. (2003). Thinking constructively with metaphors. Studies in Philosophy and Education, 22, 127-138. https://doi.org/10.1023/A:1022289113443 